
\title{
25 Research Square \\ Sox14 is essential for initiation of interneuron differentiation in the chick spinal cord
}

\section{Taiki Katsuyama}

Nara Institute of Science and Technology: Nara Sentan Kagaku Gijutsu Daigakuin Daigaku

\section{Minori Kadoya}

Nara Institute of Science and Technology: Nara Sentan Kagaku Gijutsu Daigakuin Daigaku

Manabu Shirai

National Cerebral and Cardiovascular Center: Kokuritsu Junkankibyo Kenkyu Center

Noriaki Sasai ( $\nabla$ noriakisasai@bs.naist.jp )

Nara Institute of Science and Technology: Nara Sentan Kagaku Gijutsu Daigakuin Daigaku https://orcid.org/0000-0003-0360-1138

\section{Research}

Keywords: Nerual Tube, Progenitor Cells, Postmitotic Neurons, Sox Transcription Factors, V2a region

Posted Date: February 26th, 2021

DOI: https://doi.org/10.21203/rs.3.rs-243180/v1

License: (1) (1) This work is licensed under a Creative Commons Attribution 4.0 International License. Read Full License 
1

2

3

4

5

6

7

8

9

10

11

12

13

14

15

16

17

18

19

20

5

17

\section{Sox14 is essential for initiation of interneuron differentiation} in the chick spinal cord

\footnotetext{
Taiki Katsuyama ${ }^{1}$, Minori Kadoya ${ }^{1}$, Manabu Shirai ${ }^{2}$ and Noriaki Sasai ${ }^{1 *}$

${ }^{1}$ Developmental Biomedical Science, Nara Institute of Science and Technology, 8916-5, Takayamacho, Ikoma 630-0192, Japan

${ }^{2}$ Omics Research Center (ORC), National Cerebral and Cardiovascular Center, 6-1 Kishibe Shinmachi, Suita, Osaka 564-8565, Japan

*Author to whom correspondence should be addressed.

e-mail: noriakisasai@bs.naist.jp
} 


\section{Abstract}

The neural tube comprises several different types of progenitors and postmitotic neurons that co-ordinately act with each other to play integrated functions. Its development consists of two phases: proliferation of progenitor cells and differentiation into postmitotic neurons. How progenitor cells differentiate into each corresponding neuron is an important question for understanding the mechanisms of neuronal development.

Here we introduce one of the Sox transcription factors, Sox14, which plays an essential role in the promotion of neuronal differentiation. Sox 14 belongs to the SoxB subclass and its expression starts in the progenitor regions before neuronal differentiation is initiated at the trunk level of the neural tube. After neuronal differentiation is initiated, Sox 14 expression gradually becomes confined to the V2a region of the neural tube, where Chx10 is co-expressed. Overexpression of Sox14 restricts progenitor cell proliferation. Conversely, the blockade of Sox 14 expression by the $R N A i$ strategy inhibits V2a neuron differentiation and causes expansion of the progenitor domain. We further found that Sox 14 acted as a transcriptional activator. Taken together, Sox14 acts as a modulator of cell proliferation and an initiator protein for neuronal differentiation in the intermediate region of the neural tube.

Abbreviations

38 HH stage: Hamburger and Hamilton's stage

39 pMN: motor neuron progenitor region

40 FP: floor plate

41 hpt: hours post transfection

42 sh-Sox14: short-hairpin RNA targeting Sox14

43 IdU: 5-Iodo-2'-deoxyuridine

44 Shh: Sonic Hedgehog

45 RT-qPCR: reverse transcription and quantitative polymerase chain reaction

46 TUNEL: TdT-mediated dUTP nick-end labelling 
The neural tube is the embryonic organ of the central nervous system, and several distinct types of progenitor cells and neurons are arrayed along the dorsal-ventral axis in an orderly manner [1]. The cells of each progenitor domain differentiate into their corresponding neurons, and the combination of different types of neurons exerts integrated functions as a whole [2].

Neural tube development is largely divided into two phases: the assignment and proliferation of neural progenitor cells and their differentiation into postmitotic functional neurons $[3,4]$.

During the first phase, at the trunk level of the neural tube, a variety of neural progenitor cells are patterned depending on the positional information provided by the signal molecules, collectively called morphogens. In the ventral part of the neural tube, the assignment of the neural domains is mainly governed by sonic hedgehog (Shh), which is expressed in the notochord and later in the floor plate (FP) cells. The ventral neural tube is consequently divided into six regions: $\mathrm{p} 0-\mathrm{p} 2$, motor neuron progenitor region ( $\mathrm{pMN}), \mathrm{p} 3$, and the $\mathrm{FP}$, along the intermediate region to the ventral, whose assignment is dependent on the concentration of Shh. The cells proliferate to specific numbers in a region-specific manner $[5,6]$.

During the second phase of neural tube development, progenitor cells differentiate into postmitotic neurons. This process involves proneural genes, a group of basic helix-loop-helix (bHLH) transcription factors, including neurogenins, Atoh (atonal homolog), and NeuroDs, whose expression is regulated by the Notch/Delta and retinoic acid signals [7, 8]. Combination of the transcription factors of proneural genes, including position-specific transcription factors, promote differentiation $[9,10]$. Consequently the transcription factors that characterise each neuronal region start to be expressed and each region is established. For instance, the homeodomain transcription factor Chx 10/Vsx 2 is expressed and is required for the V2a interneuron. In the absence of $\mathrm{Chx} 10$, progenitor cells acquire different neuronal identities of the motor neuron $(\mathrm{MN})$, which is located in the adjacent domain of the neural tube $[11,12]$. As such, there are many transcription factors that regulate neuronal differentiation and/or acquisition of specific functions. Important questions are how each transcription factor functions and how the gene regulatory networks are formed among them.

Among the transcription factors expressed in specific neuronal regions, we focused on one of the Sry-type transcription factors, Sox14. Sox14 belongs to the SoxB subclass, encompassing Sox1-3, Sox14, and Sox21 $[13,14]$. In previous studies, the expression of Sox14 has been shown in the V2a region, accompanied by that of Chx10, Lhx3, and Sox 21 in the developing spinal cord area, and this profile is supported by single-cell transcriptomic analysis $[15,16]$. Sox14 is also expressed in a part of the diencephalon, called the subcortical visual shell, and is required for normal circadian rhythm [17]. In humans, mutations in the Sox 14 gene cause congenital disorders, including deformation of the eyelids and severe mental retardation $[18,19]$. However, the functions of Sox 14 in the spinal cord are not fully understood. 
84 tube development. Analyses revealed that Sox14 is expressed not only during neuronal differentiation 85 stages, but also in the earlier stages, and it regulates cell cycle progression and neuronal differentiation.

86 Together, we suggest that various transcription factors share functions during differentiation into 87 specific neurons of the V2a region. 
Sox14 expression begins before neuronal differentiation starts, and its expression is gradually confined to the intermediate neuronal domains

During analysis of genes that induce domain-specific neuronal differentiation [1], we focused on the Sry-type transcription factor Sox14. Sox 14 is reportedly expressed in the V2a ventral neuronal domain $[11,20]$, and acts downstream of the homeodomain transcription factor Vsx2/Chx10 [11].

As most transcription factor expression starts after proneural gene expression is initiated, we attempted to identify the onset of Sox14 expression. We performed in situ hybridisation analysis of sections of the neural tube at various embryo stages. As a result, we found that Sox 14 is already expressed in the neural tube at HH stage 14 (Fig. 1a) before neuronal differentiation starts. Moreover, higher expression was observed in the intermediate region of the neural tube. Once neuronal differentiation started, Sox14 expression became confined to the intermediate regions (Fig. 1b), and was found in the V2 region at $\mathrm{HH}$ stage 22 (Fig. 1c).

Next, we investigated when ChxlO expression began. Chxl0 expression was not observed before the initiation of neuronal differentiation (Fig. 1d). The initial expression was found at the V2a region after neuronalisation started (Fig. 1e), and a stronger expression was found at HH stage 22 (Fig. 1f). These findings suggest that Sox14 has upstream factors other than Chx10 for its initial expression [11].

As Sox14 encodes a member of the SoxB subfamily, the Sox14 RNA probe for in situ hybridisation might have been crossed with other SoxB family members. To examine this possibility and to quantitatively evaluate the expression, we conducted an intermediate neural explant assay using the intermediate region of the neural plate. The explants were treated with different concentrations of Shh, and gene expression was evaluated using RT-qPCR every $12 \mathrm{~h}$. As a result, Sox14 was already expressed at $12 \mathrm{~h}$ in the presence of a low concentration of $\mathrm{Shh}\left(\mathrm{Shh}^{\mathrm{L}}\right.$; see Methods for the definition), and the expression increased continuously (Fig. 1g). In contrast, in the presence of a high concentration of Shh $\left(\mathrm{Shh}^{\mathrm{H}}\right)$, Soxl4 expression was not induced (Fig. 1g), supporting the finding that Soxl4 is expressed in the intermediate region of the neural tube, as obtained by in situ hybridisation (Fig. 1b).

The same concentration of Shh was used to induce Chx10; however, expression began $24 \mathrm{~h}$ after the start of culture, suggesting that the onset of Chx10 expression was later than that of Sox14 (Fig. 1h). Therefore, Soxl4 and Chxl0 are expressed in the same lineage of cells; however, Soxl4 expression precedes that of Chxl0.

Taken together, the collective expression profiling analyses suggest that the two transcription factors, Sox14 and Chx10, have distinct regulation of their expression. postmitotic states 
Next, we attempted to determine the activity of Sox 14 in neural tube development. For this purpose, we performed a forced expression of the control vector or the expression plasmid conveying the Sox 14 gene by electroporation in the neural tube, and analysed its effects at $48 \mathrm{~h}$ post-transfection

127

Results indicated that expression of Sox2, a neural progenitor marker, was reduced in the electroporated cells, while no change was found by electroporation of the control vector (Fig. 2a-b'; $\mathrm{n}=5$ for control and $\mathrm{n}=8$ for Sox 14 ). We reasoned that the cells might have precociously differentiated into neurons; however, Sox 14 overexpression also reduced $\mathrm{p} 27^{\mathrm{KIP} 1}$ expression, suggesting that neuronal maturation was also perturbed by Sox14 overexpression (Fig. 2c-d'). Therefore, we hypothesised that the initial stage of the neurons was induced by Sox 14 , and investigated the expression of $p 57^{K I P 2}$, which appears immediately after neuronal birth [21,22], using in situ hybridisation. As a result, we found the expressing area expanded into the progenitor regions, as well as the upregulated expression level (Fig. 2e-f'; $\mathrm{n}=5$ for control and n=6/8 for Sox 14).

To quantify the induction of genes involved in neurogenesis $[9,10]$, we analysed the expression of genes involved in neurogenesis using RT-qPCR in explants electroporated with Sox14. We observed selective expression of $p 57^{K I P 2}$ through the overexpression of Sox 14 , whereas other proneural genes, including Ngn1, Ngn2, and NeuroD4, remained unchanged in their expression (Fig. 2g).

Taken together, Sox 14 induces $p 57^{K I P 2}$-expressing early-stage neurons.

\section{Sox14 restricts proliferation of neural progenitor cells}

We found that the cell number tended to be reduced by Sox14 overexpression (Fig. $\left.2 \mathrm{~b}, \mathrm{~b}^{\prime}, \mathrm{d}, \mathrm{d}^{\prime}, \mathrm{f}, \mathrm{f}^{\prime}\right)$, and supposed that this may be due to the blockade of cell proliferation or programmed cell death. To test these possibilities, we performed a 5-Iodo-2'-deoxyuridine (IdU) incorporation assay to label S-phase cells at $24 \mathrm{hpt}$ of control or Sox14. As a result, the number of cells in the S-phase was greatly reduced in the Sox 14-electroporated side (Fig. 3a-c; $n=5$ for control, $n=6$ for Sox 14). In addition, immunofluorescence with phospho-histone $3(\mathrm{pHH} 3)$ to identify the M-phase cells revealed a dramatically reduced number of pHH3-positive cells in the electroporated side (Fig. 3d-f; n=5 for control, $\mathrm{n}=6$ for Sox 14 ).

Next, we investigated whether programmed cell death occurs in the Sox14-electroporated cells and performed a TdT-mediated dUTP nick-end labelling (TUNEL) assay, which detects fragmented genomic DNA. However, we did not find a significant increase in the positive signals in the Sox14electroporated side (Fig. 3g-h', j; $\mathrm{n}=5$ for control, $\mathrm{n}=6$ for Sox14), while Ptch $\Delta$-overexpressing neural cells showed positive signals (Fig. 3i,i',j; $n=7$ ) [23, 24], suggesting that the experiment per se was successful.

These findings indicate that cell cycle progression was blocked by Sox 14 . 


\section{Sox14 is required for neuronal differentiation}

To reveal the essential roles of Sox14 in neural tube development, we attempted to disrupt Sox14 expression using an RNA interference (RNAi) strategy [25]. We designed the sh-RNA construct targeting chick Sox14 (sh-Sox14) and electroporated either the control vector (sh-control) or the plasmid conveying sh-Sox14 into the neural tube. At $48 \mathrm{hpt}$, inhibition of Sox14 expression by sh-Sox14 was confirmed, while sh-control electroporation maintained expression (Fig. 4a-b'; none downregulated in control $(\mathrm{n}=5), 100 \%$ downregulated in sh-Sox14 $(\mathrm{n}=6))$. We further found that Chx10 expression was blocked by sh-Sox14 (Fig. 4c-d'; none downregulated in control $(\mathrm{n}=5), 100 \%$ downregulated in sh-Sox14 $(\mathrm{n}=6))$, suggesting that Sox14 is required for Chx10 expression.

In contrast, the motor neuron domain, characterised by Islet1, was not affected by sh-Sox14 (Fig. 4e-f'; n=5 for control, n=6 for sh-Sox 14), and the Sox2-expressing neural progenitor domain was laterally expanded (Fig. 4g-h'; no ectopic expression in control (n=5), 83\% displayed ectopic expression in sh-Sox14 (n=6)), suggesting that Soxl4 knockdown inhibited neuronal differentiation.

Therefore, Sox 14 is required for the progression of the neuronal differentiation, particularly for the V2a identity.

\section{Sox14 acts as a transcriptional activator}

Based on the amino acid sequences, SoxB transcription factors have been categorised into two subgroups, SoxB1 and SoxB2, where SoxB1 includes Sox1-3, while SoxB2 is composed of Sox 14 and Sox21 [13]. While Sox1-3 are transcriptional activators [26], Sox14 and Sox21 are thought to act as transcriptional repressors, as judged from their amino acid sequences [13]. Therefore, we attempted to reveal the mode of action of Sox14 and generated chimeric constructs of the DNA-binding region of Sox 14 fused either with the transactivating domain of VP16 of the herpes simplex virus (Sox14 ${ }_{D_{B D}}{ }^{-}$

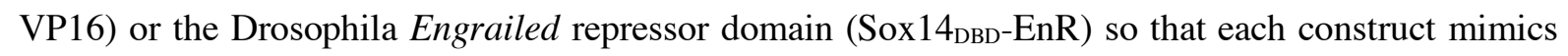
transcriptional activation or repression (Fig. 5a). By the electroporation of Sox $14_{D B D}-V P 16$, the electroporated cells abolished the expression of the neural progenitor Sox2 (Fig. 5b,b'; n=10), and $p 57^{K I P 2}$ was found to be expanded (Fig. $5 \mathrm{c}, \mathrm{c}^{\prime} ; \mathrm{n}=10$ ). Moreover, injection of IdU into the Sox $14_{D B D^{-}}$ VP16-electroporated embryos revealed that the number of S-phase cells was reduced in the

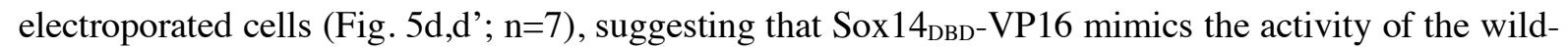
type Sox14 (Fig. 3b,c).

In contrast, the electroporation of $\operatorname{Sox} 14_{D B D}-E n R$, a mimetic construct for transcriptional repressor activity, induced the ectopic expression of Sox2 (Fig. 5e,e'; n=12) at the expense of $p 57^{K I P 2}$ (Fig. 5f,f'; n=12), suggesting that Sox $14_{\mathrm{DBD}}-\mathrm{EnR}$ exerts opposing functions compared to wild-type Sox14. In addition, the IdU injection did not change the rate of incorporation as in the control (Fig. $5 \mathrm{~g}, \mathrm{~g}^{\prime} ; \mathrm{n}=8$ ), which differs from the phenotype observed in wild-type Sox 14 (Fig. 3b,c). 
196 EnR-fused construct acts in an antimorphic manner, and (ii) Sox14 is essential for promoting the 197 differentiation of neural progenitor cells. 


\section{Sox14 expression begins earlier than initiation of neuronal differentiation.}

200

201

202

203

204

205

206

207

208

209

210

211

212

213

214

215

216

217

218

219

220

221

222

223

224

225

226

227

228

229

230

231

232

In this study, we analysed the function of one of the Sox transcription factors, Sox 14, and demonstrated that Sox 14 is required for the progression of neuronal differentiation (Fig. 4). In addition, we demonstrated that Sox 14 acts as a transcriptional activator, as the VP16-chimeric construct mimics wild-type Sox14 (Fig. 5).

Sox14 was initially identified as a SoxB subclass gene, which encompasses Sox1-3, Sox14, and Sox 21 [13]. A subsequent study demonstrated that Sox 14 expression was found in the V2a neuronal region, and its expression was overlapped, at least in part, with that of Chx10 and Lhx3/Lim3 [20]. Moreover, a recent study showed that electroporation of Chx10 induces ectopic expression of Sox14, indicating that Chx10 is a sufficient upstream factor for Sox14 [11]. In contrast, our analysis revealed that Sox 14 expression onset occurred earlier than that of Chx10 (Fig. 1), suggesting that Chx10 is not a single upstream regulator for the initiation of Sox14 expression. One possible upstream factor is retinoic acid (RA), as Sox 14 expression is upregulated by RA during neural differentiation in embryonic carcinoma cells [27]. In addition, RA has been shown to play an important role in the intermediate region of neural progenitor cells [28], and Pax6 expression was upregulated by RA, as indicated by RTqPCR analysis of neural explants. However, Sox 14 expression remained unchanged after RA treatment (Supplementary Fig. S1), suggesting that Pax6 and Sox14 expression is initiated by different mechanisms.

In addition, it is notable that there are multiple binding sites for $\mathrm{Nkx} 2.2$ and Olig2 in the regulatory region of Sox14 [29]. Considering that $\mathrm{Nkx} 2.2$ and Olig2 are induced by Shh [30] and are transcriptional repressors [31], Sox 14 may be expressed in neural progenitor cells as a default, and its expression is restricted by $\mathrm{Nkx} 2.2$ and Olig2.

\section{Sox14 is required for the progression of neuronal differentiation of intermediate neurons.}

Sox14 and Chx10 are expressed in the same region of the V2a intermediate neuronal area. Chx10 is induced by Lhx3 and consolidates V2a identity by repressing the genes that characterise the adjacent neuronal regions of non-V2a interneurons or MN [11]. Attenuation of the Chx10 gene causes aberrant upregulation of the $M N$ gene, suggesting that $\mathrm{Chx} 10$ determines the direction of neuronal identity [11].

In contrast, our analyses revealed that Sox14 is required for the promotion of neuronal differentiation. Knockdown of the Sox 14 gene caused the abolishment of neuronal differentiation, rather than producing different types of neurons (Fig. 4). Therefore, it can be said that Chx10 and Sox 14 play different functions during neuronal differentiation, Sox14 promotes differentiation, and Chx10 determines the direction. 
Previous studies have shown that Sox14 plays multiple roles in neural and neuronal 234 development. For instance, Sox14 is required for terminal differentiation of dorsal midbrain 235 GABAergic neurons [32]. In addition, Sox14 activates the p53 pathway to induce cell death in carcinoma cells [17]. While the context in which we analysed the Sox 14 function is different from these previous reports, the activities of Sox 14 revealed from our analyses are consistent with these previous results; Sox14 is required for the promotion of neuronal differentiation.

In our analyses, Sox14 acted as a transcriptional activator (Fig. 5). We recognise that Sox14 has been suggested as a transcriptional repressor, which is controversial for its mode of action. The repressor assumption was based on its amino acid sequence [13], and we predicted that the mode of action would change in a context-dependent manner.

In this study we demonstrated that Sox 14 induces the $p 57^{K I P 2}$ expression; however, whether this

244 induction is direct is still elusive. Thus future studies will focus on searching for the direct target genes 245 of Sox 14 using chromatin immunoprecipitation. Moreover, it is highly possible that the target genes of 246 Sox 14 in neural progenitor cells and those in V2a neurons are distinct. Therefore, in addition to 247 searching for different target genes depending on the neural differentiation steps, it would be useful to 248 identify cofactors that bind to Sox 14 . These cofactors would include the general proneural genes, such as Neurogenin1/2 or NeuroD2/4/6, and would modulate the functions of Sox14.

Understanding the function of each transcription factor is useful for generating specific functional neurons from stem cells [33]. We envision that the findings of this study will partly contribute to regenerative medicine in the future. 
All animal experiments were performed under the approval of national and internal legislation; approval number 1636 was obtained from the review panel of the animal experiments of the Nara Institute of Science and Technology.

258

\section{Manipulations and histological analyses on chick embryos}

Chicken eggs were purchased from Yamagishi Farm (Wakayama prefecture, Japan). The development of chick embryos was evaluated using the Hamburger and Hamilton criteria [34]. Embryos were electroporated at $\mathrm{HH}$ stage 12 with the electroporator BTX830 and were incubated at $38{ }^{\circ} \mathrm{C}$ for the indicated periods.

For the overexpression analyses, the coding regions of the genes of interest were subcloned into

265

266

267 the $p C I G$ expression vector [35], which contains the chicken $\beta$-actin promoter and the GFP gene downstream of the internal ribosomal entry site. Drosophila EnR [36] and herpes-simplex virus-derived VP16 transactivation domain [37] chimeric constructs were generated by fusing the amino-acids 1-90 of chicken Sox 14 .

To construct sh-Sox 14, the sequence AAGCCTCCGGTTGCCTACATATTAT was subcloned into the vector $p R F P R N A i$ [25], in which the electroporated cells were identified by RFP expression.

For tissue analyses, the harvested embryos were fixed with $4 \%$ paraformaldehyde for $90 \mathrm{~min}$, and were replaced with $15 \%(\mathrm{w} / \mathrm{v})$ sucrose overnight with rotating incubation. These embryos were embedded in OCT compound (Sakura) and cryosectioned to a thickness of $12 \mu \mathrm{m}$ using a Polar cryostat (Sakura Finetek). The following antibodies were used in this study: Sox2 (rabbit; Millipore, \#5603), p27 (mouse; BD Transduction Laboratories, \#610241), Olig2 (rabbit; Millipore; \#9610), Nkx2.2 (mouse; DSHB; \#74.5A5), Islet1 (mouse; DSHB; \#39.4D5), Pax7 (mouse; DSHB), and GFP (sheep; AbD Serotec; \#4745-1051). The secondary antibodies used were rabbit (Jackson Laboratories; \#711166-152 for Cy3; \#711-606-152 for Cy5), mouse (\#715-166-151 for Cy3; \#715-606-150 for Cy5), and sheep (\#713-096-147 for FITC).

In situ hybridisation and TUNEL assays were performed as previously described $[6,38,39]$. For in situ hybridisation analyses, the expression of genes or si-RNAs was validated by the tracer (GFP/RFP) expression on the adjacent sections. As a positive control for the TUNEL assay, $p C I G$ Ptch $\Delta$, encoding an insensitive mutant to the Hedgehog signal [23], was electroporated, as the blockade of the Hedgehog signal has been shown to induce programmed cell death [24].

For IdU incorporation assays, $100 \mathrm{mM}$ IdU (Wako, Japan) was injected into the neural tube cavity at $22 \mathrm{hpt}$ and incubated for $2 \mathrm{~h}$. To identify the incorporated cells, the sectioned tissues were pretreated with 1M hydrochloric acid, and the IdU antibody (mouse; Thermo; MA5-24879) was used. 


\section{Explants and RT-qPCR}

The intermediate neural explants were prepared as described previously [38, 39]. Briefly, HH stage 9 embryos were placed in L-15 medium and the caudal stem zone was excised [40]. After

292 treatment with $10 \mu \mathrm{g} / \mathrm{mL}$ dispase II (Sigma), the intermediate region of the neural plate was further cut 293 out and embedded in the collagen gel (Sigma). The embedded cells were cultured with DMEM/F-12 294 supplemented with Mitoserum extender (BD Biosciences) and 1× penicillin/streptomycin/glutamine 295 mixture (Wako, Japan). A high concentration of Shh $\left(\mathrm{Shh}^{\mathrm{H}}\right)$ was defined as the concentration at which 296 approximately $80 \%$ of $\mathrm{Nkx} 2.2$-positive cells were found, whereas a low concentration of $\mathrm{Shh}\left(\mathrm{Shh}^{\mathrm{L}}\right)$ 297 resulted in approximately $60 \%$ of Olig2-positive cells at $24 \mathrm{~h}$ [41]. For RT-qPCR, RNA was extracted 298 using the Picopure RNA extraction kit (Thermo; \#KIT0204) and analysed using a CFX qPCR machine 299 (Bio-Rad). Expression levels were normalised with that of Glyceraldehyde-3-Phosphate 300 Dehydrogenase (GAPDH). The primer sequences used are shown in Supplementary Tab. S1.

301

302 Image processing and statistics

303 Images were captured using the LSM710 confocal microscope or AxioVision (Carl Zeiss) and were 304 processed using the software Photoshop (Adobe). Figures were formed using an Illustrator (Adobe). 305 Quantitative data are presented as means \pm SEM, and differences were evaluated using the two-tailed 306 Student's $t$ test. Statistical significance was set at $p<0.05$, and $p$-values $(*<0.05 ; * * ; p<0.01$, ***; 307 $p<0.001)$ are indicated in each graph. 


\section{Ethics approval and consent to participate}

310 This study does not include human participants, tissues or data.

311

312 Consent for publication

313 Not applicable.

314

\section{Competing interests}

316 The authors declare that they have no competing interests.

317

318 Availability of data and material

319 All relevant data are presented in the text and figures. The plasmids used in this study are available 320 from the corresponding author upon request.

321

322 Funding

323 This work was supported in part by grants-in-aid for scientific research from the Japan Society for the 324 Promotion of Science (19H04781 and 20H03263) to NS, and by a Sasakawa Scientific Research Grant 325 from the Japan Science Society to MK.

326

327 Authors' contributions

328 NS conceived the project; TK, NS, MS performed experiments and analysed the data; MK provided 329 essential materials; all authors joined the discussion; NS wrote the manuscript.

331 Acknowledgements

332 The authors thank Erika Yoshihara for comments, Chikara Kogiso and Atsuki Yatsuzuka for technical 333 guidance, and all other laboratory members for their support and discussions. 


\section{Figure legends}

\section{Fig. 1 Sox14 expression begins before initiation of neuronal differentiation. (a-f) Cross-sections of} the trunk level of the neural tube at HH stages 14 (a,d), 18 (b,e), and 24 (c,f) of chick embryos were analysed either using in situ hybridisation with probes against Sox14 (a-c) and Chx10 (d-f). Expression is indicated with a bracket (b) or with arrowheads (c,e,f). Scale bar in (a) $=100 \mu \mathrm{m}$ for (a-f). (g,h) Expression analysis of Sox 14 (g) and Chx10 (h) using RT-qPCR on the explants cultured in the control medium, or with $\mathrm{Shh}^{\mathrm{L}}$ or $\mathrm{Shh}^{\mathrm{H}}$ for various time periods.

Fig. 2 Sox14 induces early neuronal differentiation during the neural tube development. $p C I G$ (a,a',c,c',e,e') or pCIG-Soxl4 (b,b',d,d',f,f') were electroporated at HH stage 12 and embryos were harvested at 48 hpt. Expression levels of Sox2 (red; a-b') and p2 $7^{\text {KIP1 }}$ (red; c-d') were analysed using immunofluorescence, and the $p 57^{K I P 2}$ (e-f') was analysed using in situ hybridisation. Scale bar in (a) = $50 \mu \mathrm{m}$ for (a-f'). (g) The effect of the electroporation of $p C I G$ or $p C I G-S o x 14$ was analysed using RTqPCR at 36 hpt on the explants electroporated with $p C I G$ (blue bars) or $p C I G-S o x 14$ (red bars). Note that, in (e', $\left.\mathrm{f}^{\prime}\right)$, the GFP expression was taken from the adjacent sections.

Fig. 3 Sox14 inhibits cell proliferation without inducing programmed cell death. Either $p C I G$ (control; a,a',c,d,d',f,g,g',j) or $p C I G-S o x 14$ (b,b',c,e,e',f,h,h',j) were electroporated into HH stage 12 and embryos were subjected to the following assays. (a-c) IdU incorporation assay to detect the S-phase cells. IdU was injected into the cavity of the neural tube at $22 \mathrm{hpt}$ for 2 hours, and embryos were harvested for immunofluorescence with the IdU antibody. (c) Quantification of the IdU-positive cells in the total GFP-positive cells. (d-f) Immunofluorescence with the M-phase marker phospho-Histone 3 (pHH3). Embryos were harvested at $24 \mathrm{hpt}$ and were subject to immunofluorescence with pHH3 antibody. (f) Quantification of the pHH3-positive cells over the GFP-positive cells on the apical side. (g-j) TUNEL assay to detect programmed cell death. Embryos were harvested at $24 \mathrm{hpt}$ as in (d-f) and were analysed using a TUNEL assay. (i,i') For the positive control of the TUNEL assay, pCIG-Ptch $\Delta$ was electroporated and the same assay as in (g-h') was performed. (j) Quantification of the TUNELpositive cells in all GFP-positive cells. Scale bar in (a) $=50 \mu \mathrm{m}$ for (a-b',d-e',g-i').

Fig. 4 Sox14 is required for neuronal differentiation. Embryos were electroporated either with $p R N A i$ (a,a',c,c',e,e', g,g') or $p R N A i-S o x 14$ (b,b',d,d',f,f',h,h') and were analysed at 48 hpt. Sox14 (ab') and Chx10 (c-d') expression levels were analysed using in situ hybridisation, and Islet1 (e-f') and Sox2 (g-h') expression was analysed using immunofluorescence. Normal (a,b,c,d) or ectopic (h) expression is indicated by filled arrowheads; reduced expression $(b, d)$ by outlined arrowheads. Scale bars in (a) for (a-d') and in (e) for (e-h') $=50 \mu \mathrm{m}$. Note that the RFP expression in (a', b', c', d') was taken from the adjacent sections. 
371 Fig. 5 Sox14 acts as a transcriptional activator. (a) Schematic of the Sox $14_{\mathrm{DBD}}-\mathrm{VP} 16$ and Sox $14_{\mathrm{DBD}}$

372 EnR. DBD; DNA-binding domain of Sox14 (amino acid numbers 1-90 of chicken Sox14). (b-h) Either

$373 p C I G-S o x 14_{D B D}-V P 16$ (b-d') or $p C I G-S o x 14_{D B D}-E n R(\mathrm{e}-\mathrm{g}$ ') was electroporated into the neural tube and

374 the embryos were analysed using immunofluorescence with antibodies against Sox2 (b,b',e,e') and

$375 \mathrm{p} 57^{\mathrm{KIP} 2}$ (c, ', 'f,f') at $48 \mathrm{hpt}$, or were subjected to an IdU incorporation assay at $24 \mathrm{hpt}$, as in Fig. 3a-c.

376 Ectopic (e,f) and reduced (b,c) expression is indicated by filled and outlined arrowheads, respectively.

377 Note that the GFP expression in (c', f') was taken from the adjacent sections. Scale bars in (b) for (b-

$\left.378 \mathrm{c}^{\prime}, \mathrm{e}-\mathrm{f}^{\prime}\right)$ and in (d) for (d, d',g,g') $=50 \mu \mathrm{m}$. (h) Quantification of the IdU-positive cells in all GFP-positive

379 cells. The ratio of the control condition is taken from Fig. 3c.

380

381 Supplementary Figures

382 Supplementary Fig. S1 Sox14 expression is not upregulated by retinoic acid (RA). Intermediate

383 neural explants were cultured either in the control medium or the medium with RA (100 nM) for 24

384 hours to be subjected for an RT-qPCR.

385

386 Supplementary Table

387 Supplementary Tab. S1 The RT-qPCR primers used in this study. 
1. $\quad$ Alaynick, W.A., T.M. Jessell, and S.L. Pfaff, SnapShot: spinal cord development. Cell, 2011. 146(1): p. 178-178 e1.

2. Briscoe, J. and B.G. Novitch, Regulatory pathways linking progenitor patterning, cell fates and neurogenesis in the ventral neural tube. Philos Trans R Soc Lond B Biol Sci, 2008. 363(1489): p. 57-70.

3. Hardwick, L.J., et al., Cell cycle regulation of proliferation versus differentiation in the central nervous system. Cell Tissue Res, 2015. 359(1): p. 187-200.

4. Sasai, N., M. Kadoya, and A. Ong Lee Chen, Neural induction: Historical views and application to pluripotent stem cells. Dev Growth Differ, 2020.

5. Sagner, A. and J. Briscoe, Morphogen interpretation: concentration, time, competence, and signaling dynamics. Wiley Interdiscip Rev Dev Biol, 2017.

6. Kadoya, M. and N. Sasai, Negative Regulation of mTOR Signaling Restricts Cell Proliferation in the Floor Plate. Front Neurosci, 2019. 13: p. 1022.

7. Janesick, A., S.C. Wu, and B. Blumberg, Retinoic acid signaling and neuronal differentiation. Cell Mol Life Sci, 2015. 72(8): p. 1559-76.

8. Bertrand, N., D.S. Castro, and F. Guillemot, Proneural genes and the specification of neural cell types. Nat Rev Neurosci, 2002. 3(7): p. 517-30.

9. Guillemot, F., Spatial and temporal specification of neural fates by transcription factor codes. Development, 2007. 134(21): p. 3771-80.

10. Diez del Corral, R. and K.G. Storey, Markers in vertebrate neurogenesis. Nat Rev Neurosci, 2001. 2(11): p. 835-9.

11. Clovis, Y.M., et al., Chx10 Consolidates V2a Interneuron Identity through Two Distinct Gene Repression Modes. Cell Rep, 2016. 16(6): p. 1642-1652.

12. Debrulle, S., et al., Vsxl and Chx10 paralogs sequentially secure V2 interneuron identity during spinal cord development. Cell Mol Life Sci, 2020. 77(20): p. 4117-4131.

13. Uchikawa, M., Y. Kamachi, and H. Kondoh, Two distinct subgroups of Group B Sox genes for transcriptional activators and repressors: their expression during embryonic organogenesis of the chicken. Mech Dev, 1999. 84(1-2): p. 103-20.

14. Bylund, M., et al., Vertebrate neurogenesis is counteracted by Soxl-3 activity. Nat Neurosci, 2003. 6(11): p. 1162-8.

15. Delile, J., et al., Single cell transcriptomics reveals spatial and temporal dynamics of gene expression in the developing mouse spinal cord. Development, 2019. 146(12).

16. Sandberg, M., M. Kallstrom, and J. Muhr, Sox21 promotes the progression of vertebrate neurogenesis. Nat Neurosci, 2005. 8(8): p. 995-1001.

17. Stanisavljevic, D., et al., SOX14 activates the p53 signaling pathway and induces apoptosis in a cervical carcinoma cell line. PLoS One, 2017. 12(9): p. e0184686.

18. Hargrave, M., et al., Fine mapping of the neurally expressed gene SOX14 to human 3q23, relative to three congenital diseases. Hum Genet, 2000. 106(4): p. 432-9.

19. Wilmore, H.P., et al., SOXI4 is a candidate gene for limb defects associated with BPES and Mobius syndrome. Hum Genet, 2000. 106(3): p. 269-76.

20. Hargrave, M., et al., The HMG box transcription factor gene Sox 14 marks a novel subset of ventral interneurons and is regulated by sonic hedgehog. Dev Biol, 2000. 219(1): p. 142-53.

21. Creff, J. and A. Besson, Functional Versatility of the CDK Inhibitor p57(Kip2). Front Cell Dev Biol, 2020. 8: p. 584590.

22. Pateras, I.S., et al., p57KIP2: "Kip"ing the cell under control. Mol Cancer Res, 2009. 7(12): p. 190219.

23. Briscoe, J., et al., A hedgehog-insensitive form of patched provides evidence for direct long-range morphogen activity of sonic hedgehog in the neural tube. Mol Cell, 2001. 7(6): p. 1279-91.

24. Cayuso, J., et al., The Sonic hedgehog pathway independently controls the patterning, proliferation and survival of neuroepithelial cells by regulating Gli activity. Development, 2006. 133(3): p. 51728.

25. Das, R.M., et al., A robust system for RNA interference in the chicken using a modified microRNA operon. Dev Biol, 2006. 294(2): p. 554-63.

26. Kishi, M., et al., Requirement of Sox2-mediated signaling for differentiation of early Xenopus neuroectoderm. Development, 2000. 127(4): p. 791-800. 
27. Popovic, J., et al., Expression analysis of SOX14 during retinoic acid induced neural differentiation of embryonal carcinoma cells and assessment of the effect of its ectopic expression on SOXB

28. Novitch, B.G., et al., A requirement for retinoic acid-mediated transcriptional activation in ventral neural patterning and motor neuron specification. Neuron, 2003. 40(1): p. 81-95.

29. Kutejova, E., et al., Neural Progenitors Adopt Specific Identities by Directly Repressing All Alternative Progenitor Transcriptional Programs. Dev Cell, 2016. 36(6): p. 639-53.

30. Balaskas, N., et al., Gene regulatory logic for reading the Sonic Hedgehog signaling gradient in the vertebrate neural tube. Cell, 2012. 148(1-2): p. 273-84.

31. Zhou, Q., G. Choi, and D.J. Anderson, The bHLH transcription factor Olig2 promotes oligodendrocyte differentiation in collaboration with Nkx2.2. Neuron, 2001. 31(5): p. 791-807.

32. Makrides, N., et al., Sequential Role of SOXB2 Factors in GABAergic Neuron Specification of the Dorsal Midbrain. Front Mol Neurosci, 2018. 11: p. 152.

33. Butts, J.C., et al., Differentiation of V2a interneurons from human pluripotent stem cells. Proc Natl Acad Sci U S A, 2017. 114(19): p. 4969-4974.

34. Hamburger, V. and H.L. Hamilton, A series of normal stages in the development of the chick embryo. 1951. Dev Dyn, 1992. 195(4): p. 231-72.

35. Megason, S.G. and A.P. McMahon, A mitogen gradient of dorsal midline Wnts organizes growth in the CNS. Development, 2002. 129(9): p. 2087-98.

36. Smith, S.T. and J.B. Jaynes, A conserved region of engrailed, shared among all en-, gsc-, Nk1-, Nk2- and msh-class homeoproteins, mediates active transcriptional repression in vivo. Development, 1996. 122(10): p. 3141-50.

37. Triezenberg, S.J., R.C. Kingsbury, and S.L. McKnight, Functional dissection of VP16, the transactivator of herpes simplex virus immediate early gene expression. Genes Dev, 1988. 2(6): p. 71829.

38. Sasai, N., E. Kutejova, and J. Briscoe, Integration of signals along orthogonal axes of the vertebrate neural tube controls progenitor competence and increases cell diversity. PLoS Biol, 2014. 12(7): p. e1001907.

39. Ribes, V., et al., Distinct Sonic Hedgehog signaling dynamics specify floor plate and ventral neuronal progenitors in the vertebrate neural tube. Genes Dev, 2010. 24(11): p. 1186-200.

40. Delfino-Machin, M., et al., Specification and maintenance of the spinal cord stem zone. Development, 2005. 132(19): p. 4273-83.

41. Yatsuzuka, A., et al., GPR17 is an essential regulator for the temporal adaptation of sonic hedgehog signalling in neural tube development. Development, 2019. 146(17). 

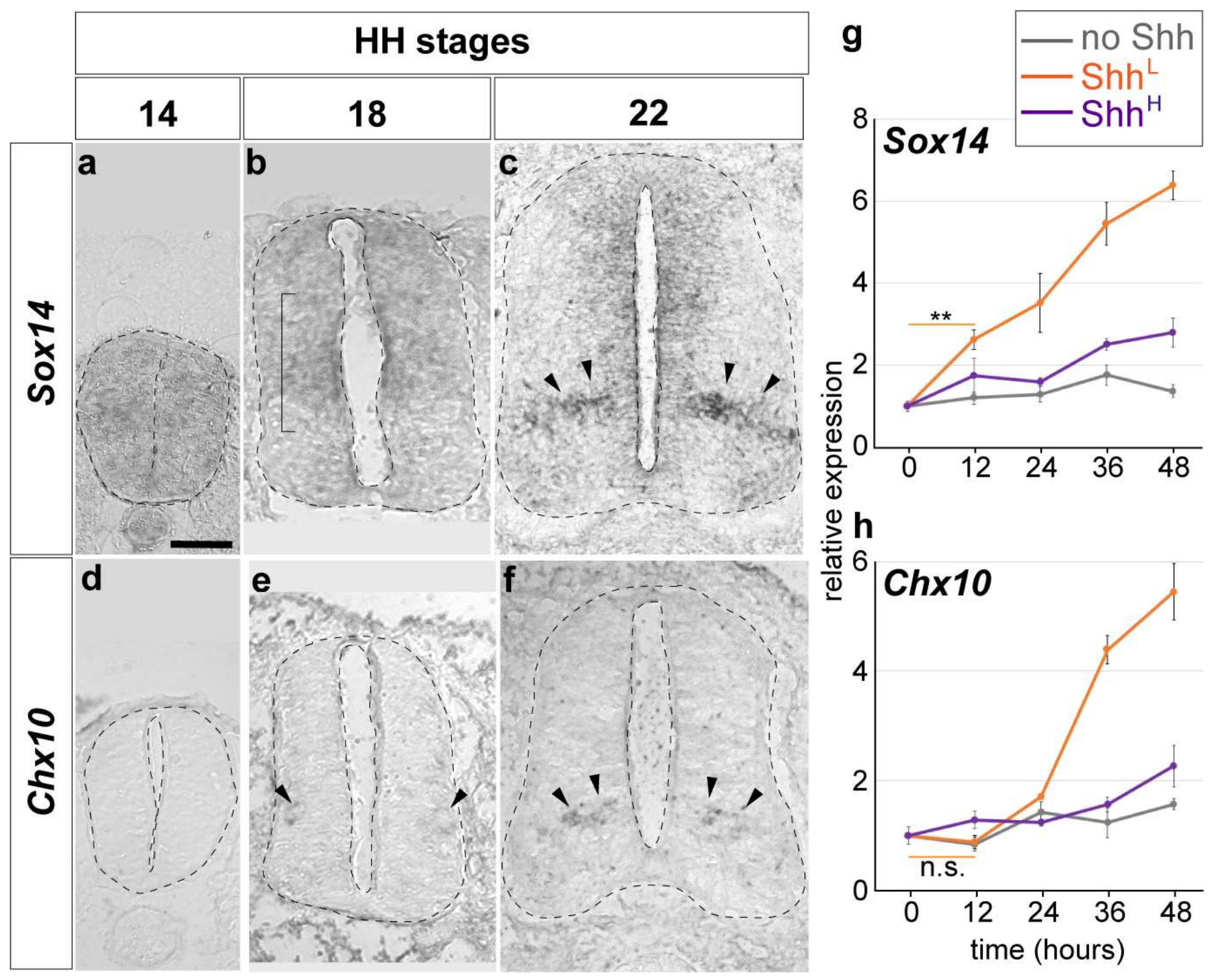

Figure 1

Sox14 expression begins before initiation of neuronal differentiation. (a-f) Cross-sections of the trunk level of the neural tube at HH stages $14(a, d), 18(b, e)$, and $24(c, f)$ of chick embryos were analysed either using in situ hybridisation with probes against Sox14 (a-c) and Chx10 (d-f). Expression is indicated with a bracket (b) or with arrowheads (c,e,f). Scale bar in (a) $=100 \mu \mathrm{m}$ for (a-f). (g,h) Expression analysis of Sox14 (g) and Chx10 (h) using RT-qPCR on the explants cultured in the control medium, or with ShhL or ShhH for various time periods. 

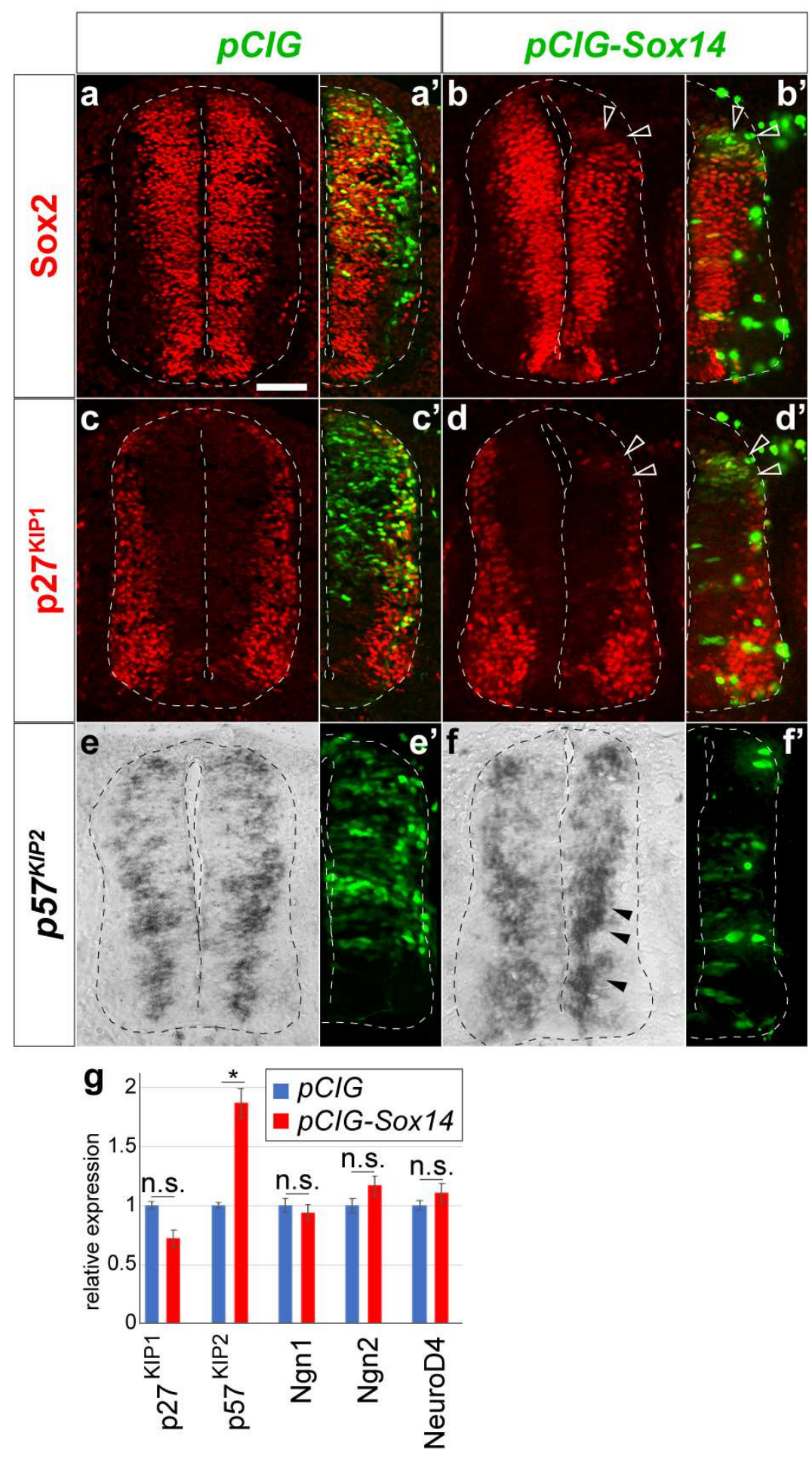

\section{Figure 2}

Sox14 induces early neuronal differentiation during the neural tube development. pCIG $\left(a, a^{\prime}, c, c^{\prime}, e, e^{\prime}\right)$ or pCIG-Sox14 (b,b',d,d',f,f') were electroporated at HH stage 12 and embryos were harvested at $48 \mathrm{hpt}$. Expression levels of Sox2 (red; a-b') and p27KIP1 (red; c-d') were analysed using immunofluorescence, and the p57KIP2 (e-f') was analysed using in situ hybridisation. Scale bar in (a) $=50 \mu \mathrm{m}$ for (a-f'). (g) The effect of the electroporation of pCIG or pCIG-Sox14 was analysed using RT qPCR at $36 \mathrm{hpt}$ on the 
explants electroporated with pCIG (blue bars) or pCIG-Sox14 (red bars). Note that, in (e', f'), the GFP expression was taken from the adjacent sections.

\section{C}

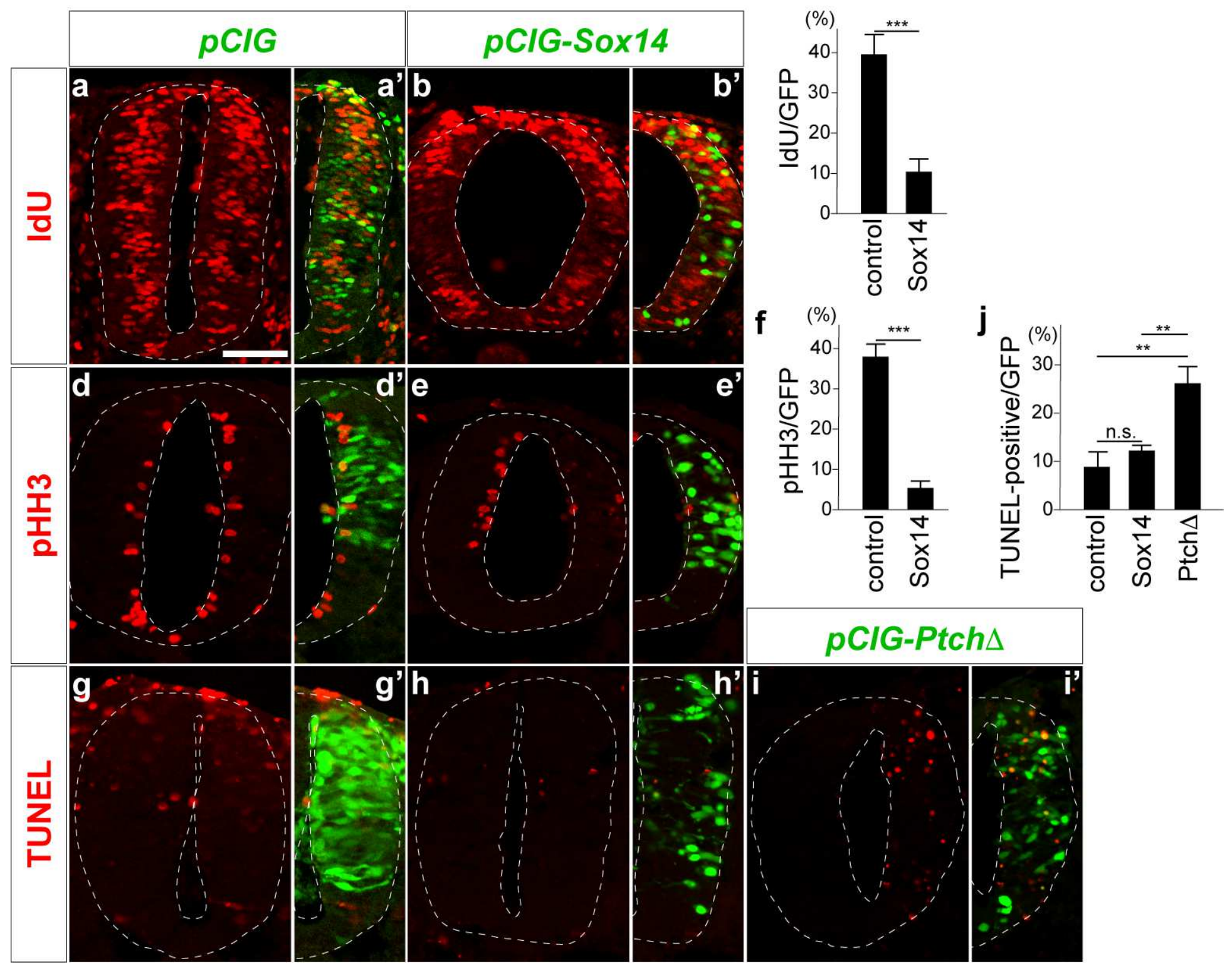

Figure 3

Sox14 inhibits cell proliferation without inducing programmed cell death. Either PCIG (control; a,a',c,d,d',f,g,g',j) or pClG-Sox14 (b,b',c,e,e,'f,h,h',j) were electroporated into HH stage 12 and embryos were subjected to the following assays. (a-c) IdU incorporation assay to detect the S-phase cells. IdU was injected into the cavity of the neural tube at $22 \mathrm{hpt}$ for 2 hours, and embryos were harvested for immunofluorescence with the IdU antibody. (c) Quantification of the IdU-positive cells in the total GFPpositive cells. (d-f) Immunofluorescence with the M-phase marker phospho-Histone 3 (pHH3). Embryos were harvested at $24 \mathrm{hpt}$ and were subject to immunofluorescence with pHH3 antibody. (f) Quantification of the pHH3-positive cells over the GFP-positive cells on the apical side. (g-j) TUNEL assay to detect programmed cell death. Embryos were harvested at $24 \mathrm{hpt}$ as in (d-f) and were analysed using a TUNEL 
assay. (i,i') For the positive control of the TUNEL assay, pCIG-PtchD was electroporated and the same assay as in (g-h') was performed. (j) Quantification of the TUNEL positive cells in all GFP-positive cells. Scale bar in $(a)=50 \mu \mathrm{m}$ for $\left(a-b^{\prime}, d-e^{\prime}, g-i^{\prime}\right)$.

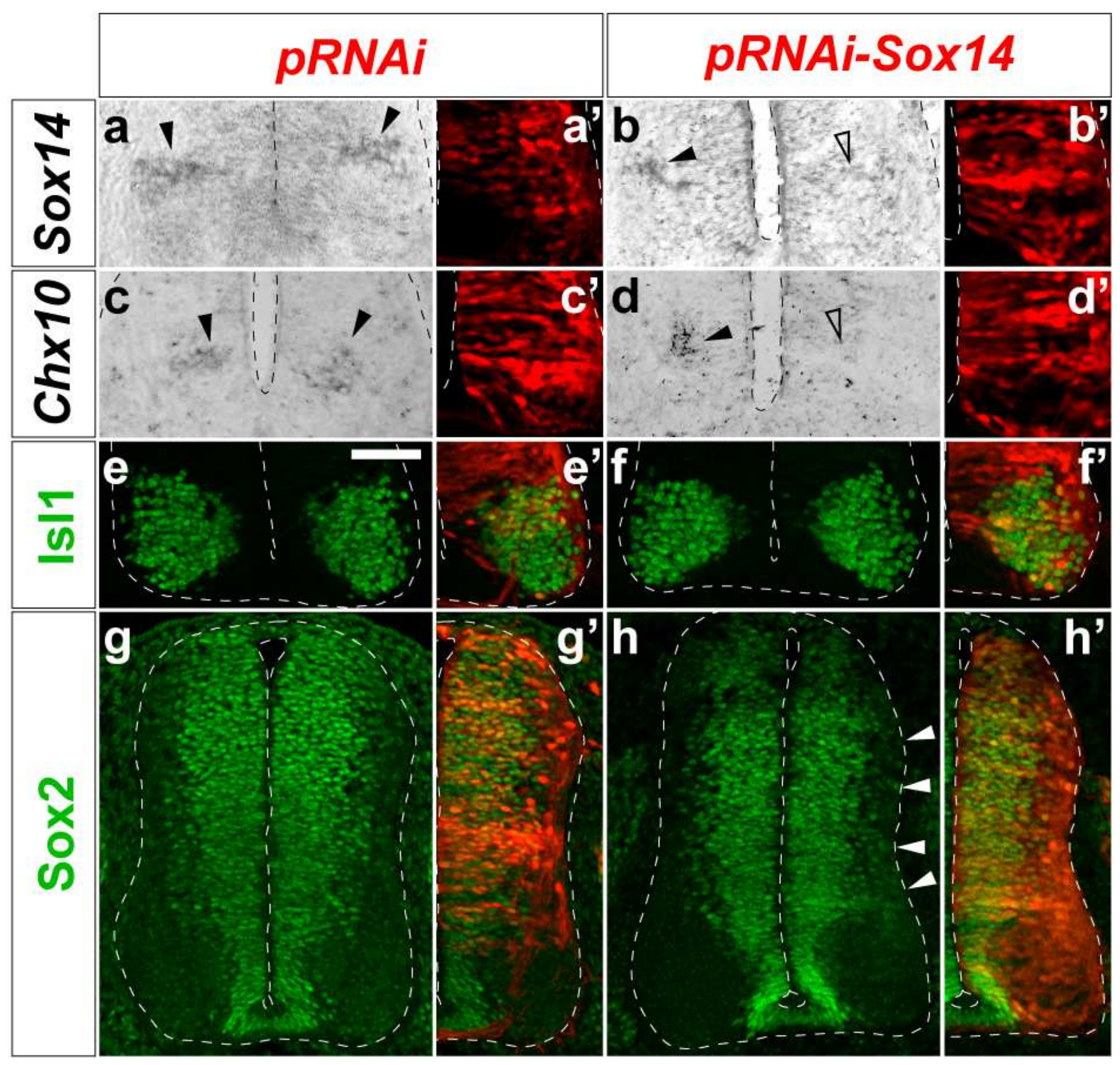

Figure 4

Sox14 is required for neuronal differentiation. Embryos were electroporated either with pRNAi $\left(\mathrm{a}, \mathrm{a}^{\prime}, \mathrm{c}, \mathrm{c}^{\prime}, \mathrm{e}, \mathrm{e}^{\prime}, \mathrm{g}, \mathrm{g}^{\prime}\right)$ or pRNAi-Sox14 (b,b',d,d',f, $\left.\mathrm{f}^{\prime}, \mathrm{h}, \mathrm{h}^{\prime}\right)$ and were analysed at $48 \mathrm{hpt}$. Sox14 (a b') and Chx10 (c- 
$\left.d^{\prime}\right)$ expression levels were analysed using in situ hybridisation, and Islet1 (e-f') and Sox2 ( $\left.\mathrm{g}-\mathrm{h}^{\prime}\right)$ expression was analysed using immunofluorescence. Normal $(a, b, c, d)$ or ectopic $(h)$ expression is indicated by filled arrowheads; reduced expression (b,d) by outlined arrowheads. Scale bars in (a) for (a-d') and in (e) for (e$\left.h^{\prime}\right)=50 \mu \mathrm{m}$. Note that the RFP expression in $\left(a^{\prime}, b^{\prime}, c^{\prime}, d^{\prime}\right)$ was taken from the adjacent sections.
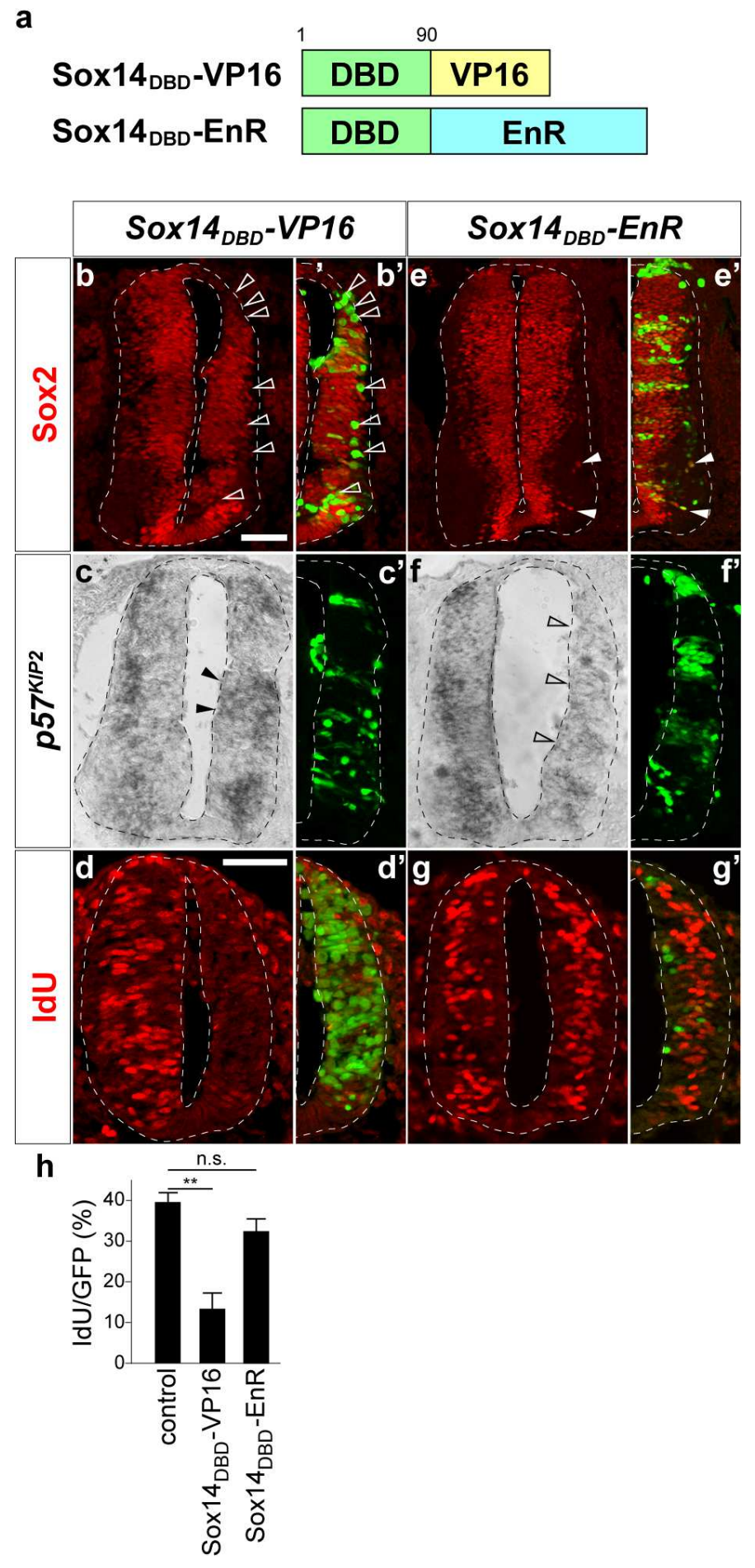

Figure 5 
Sox14 acts as a transcriptional activator. (a) Schematic of the Sox14DBD-VP16 and Sox14DBD EnR. DBD; DNA-binding domain of Sox14 (amino acid numbers 1-90 of chicken Sox14). (b-h) Either pCIGSox14DBD-VP16 (b-d') or pCIG-Sox14DBD-EnR (e-g') was electroporated into the neural tube and the embryos were analysed using immunofluorescence with antibodies against Sox2 (b,b',e,e') and p57KIP2 $\left(c, c^{\prime}, f, f^{\prime}\right)$ at $48 \mathrm{hpt}$, or were subjected to an IdU incorporation assay at $24 \mathrm{hpt}$, as in Fig. 3a-c. Ectopic (e,f) and reduced $(b, c)$ expression is indicated by filled and outlined arrowheads, respectively. Note that the GFP expression in (c', $\left.f^{\prime}\right)$ was taken from the adjacent sections. Scale bars in (b) for (b c',e-f') and in (d) for $\left(d, d^{\prime}, g, g^{\prime}\right)=50 \mu \mathrm{m}$. (h) Quantification of the IdU-positive cells in all GFP-positive cells. The ratio of the control condition is taken from Fig. 3c.

\section{Supplementary Files}

This is a list of supplementary files associated with this preprint. Click to download.

- KatsuyamaSupplFig.S1.tif

- KatsuyamaSupplementaryTableS1.jpg 